Check for updates

Cite this: Chem. Commun., 2019, 55, 2293

Received 12th December 2018, Accepted 28th January 2019

DOI: $10.1039 / \mathrm{c} 8 \mathrm{cc} 09853 \mathrm{k}$

rsc.li/chemcomm

A simple and air-stable copper( $(1) / \mathrm{N}$-heterocyclic carbene complex enables the catalytic hydrogenation of enoates and enamides, hitherto unreactive substrates employing homogeneous copper catalysis and $\mathrm{H}_{2}$ as a terminal reducing agent. This atom economic transformation replaces commonly employed hydrosilanes and can also be carried out in an asymmetric fashion.

One of the main challenges of contemporary method development for synthetic chemistry is the development of atom economic and sustainable transformations. ${ }^{1,2}$ In this vein, catalytic hydrogenations are much desired reactions, as they serve to replace complex and waste-generating reducing agents such as borohydrides, aluminium hydrides or hydrosilanes. ${ }^{3,4}$ Catalytic reactions involving copper hydride intermediates ${ }^{5}$ serve as a prime example for this challenge: commonly, hydrosilanes are used as stoichiometric reducing agents, whereas the use of dihydrogen $\left(\mathrm{H}_{2}\right)$ for the considerably more atom economic - generation of the desired copper hydride complexes has been much less explored. ${ }^{6,7}$ The hallmark reaction of the so-called copper hydride catalysis ${ }^{5}$ is arguably the conjugate reduction of $\alpha, \beta$-unsaturated carbonyl or carboxyl compounds employing hydrosilanes (Scheme 1a). Even though the replacement of hydrosilanes with $\mathrm{H}_{2}$ has been attempted for the reduction of enones, the chemoselectivity of the 1,2- vs. the 1,4-reduction turned out to be challenging to control (Scheme 1b). ${ }^{6}$ Furthermore, the corresponding enoates emerged as too unreactive in combination with $\mathrm{H}_{2}{ }^{8,9}$ Enoates are common substrates for the catalytic hydrogenation with precious metals such as rhodium and ruthenium, ${ }^{3,10}$ and replacement with base metal catalysts such as copper complexes is highly desirable.

We herein report the first copper-catalysed conjugate reduction of previously unreactive $\alpha, \beta$-unsaturated esters and amides with $\mathrm{H}_{2}$ employing well-defined and air-stable copper(I)/N-heterocyclic

Institut für Chemie, Technische Universität Berlin, Strasse des 17. Juni 115, 10623 Berlin, Germany. E-mail: johannes.teichert@chem.tu-berlin.de $\dagger$ Electronic supplementary information (ESI) available: Experimental procedures, characterisation and NMR spectra. See DOI: 10.1039/c8cc09853k

\section{previous work:}

catalytic conjugate reduction of enones and enoates

with hydrosilanes (generates Si-based waste)

a)

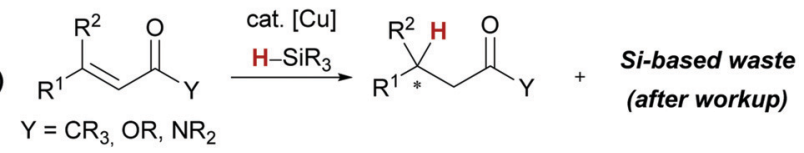

catalytic hydrogenation of enoates (chemoselectivity issues)

b)

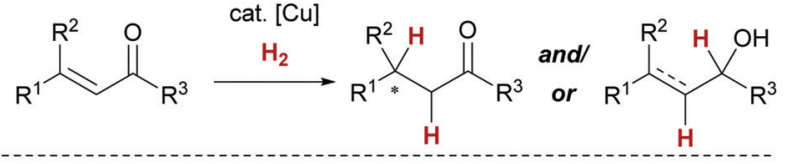

This work: catalytic hydrogenation of enoates with well-defined copper/NHC complexes c)

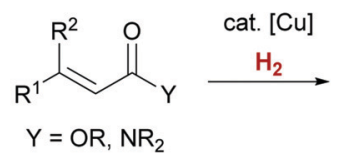

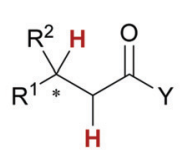

- simple protocol

- selective

- waste free

- air-stable catalyst
Scheme 1 Challenges in atom-economic copper-catalysed conjugate reductions.

carbene complexes ${ }^{11}$ (Scheme 1c). Additionally, this approach circumvents the use of waste-generating hydrosilanes, resulting in a simple and sustainable protocol for the catalytic hydrogenation of $\alpha, \beta$-unsaturated esters and amides.

The copper-catalysed hydrogenation of enoates was optimised employing ethyl $\beta$-methyl cinnamate (1, Table 1). Using sodium tert-butanolate $(\mathrm{NaO} \mathrm{Bu})$ as additive for the generation of the key $\mathrm{Cu}$-O-bond for $\mathrm{H}_{2}$ activation, ${ }^{12}$ we investigated common copper(I)/ NHC complexes 3-7 at 90 bar $\mathrm{H}_{2}$ and $60{ }^{\circ} \mathrm{C}$ in THF (Table 1, entries 1-5). From these experiments, mesityl-derived complexes $\mathbf{3}$ and $\mathbf{6}$ emerged as most active, as judged by the conversion of $\mathbf{1}$ to 2. Notably, no other side-products were observed. We found that the amount of $\mathrm{NaO} t \mathrm{Bu}$ could be lowered to $30 \mathrm{~mol} \%$ while maintaining full conversion, however, even smaller amounts of the additive $(10 \mathrm{~mol} \%)$ led to an almost complete halt of the reaction (Table 1, entries 6 and 7). Lowering the $\mathrm{H}_{2}$ pressure to $50 \mathrm{bar}$ at these limiting conditions led to diminished conversion 
Table 1 Cu-catalysed hydrogenation of enoates, optimisation ${ }^{a}$

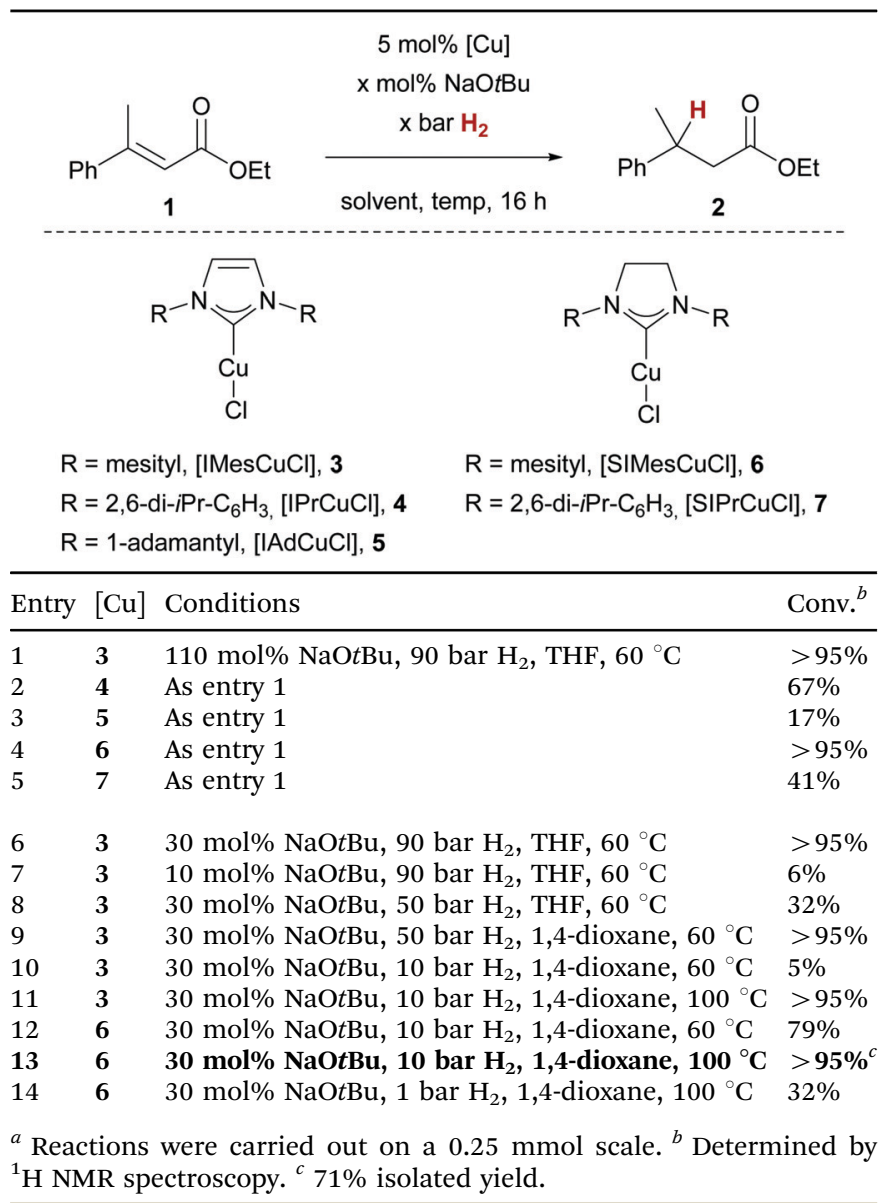

of $32 \%$ (Table 1 , entry 8 ). A subsequent solvent optimisation revealed 1,4-dioxane as optimal, ${ }^{13}$ with full conversion of 1 reached at lowered $\mathrm{H}_{2}$ pressure of 50 bar (Table 1, entry 9). When comparing catalysts 3 and 6 at 10 bar $\mathrm{H}_{2}$ pressure, imidazolinium-based copper complex 6 turned out to be more active (Table 1, entries 10 vs. 12), even though no reactivity difference could be detected at higher temperature of $100{ }^{\circ} \mathrm{C}$. The investigation of the substrate scope was therefore carried out with catalyst 6. Notably, lowering the $\mathrm{H}_{2}$ pressure with catalyst 6 to 1 bar still led to a detectable conversion of 1 ( $32 \%$ conv. Table 1 , entry 14$)$.

With optimised reaction conditions in hand, we set out to investigate the substrate scope of the copper-catalysed conjugate reduction of $\alpha, \beta$-unsaturated carboxylic acid derivatives 8 (Scheme 2). We found that the catalytic hydrogenation could be applied to a variety of aryl or alkyl substituted enoates 8. Next to the successful generation of simple naphthyl derivate $\mathbf{9 a}$, also the sterically more demanding tert-butyl ester $\mathbf{9 b}$ as well as diphenyl-substituted ester $\mathbf{9 c}$ could be furnished in good yields (68-84\%). Both electron donating and withdrawing groups were tolerated as substituents of the cinnamic acid derivatives 9d-9h with similar results in terms of yield. Notably, no protodehalogenation was observed with bromide 9f or chloride $9 \mathrm{~g}$. As in previous studies, ${ }^{7}$ the presence of a nitro or a carbonyl group (9i, $\mathbf{9 k}$ ) led to no or diminished conversion, whereas nitrile derivative $\mathbf{9 j}$ led to full conversion. Of note is the fact that protic groups such as a free phenol (91) were susceptible to the

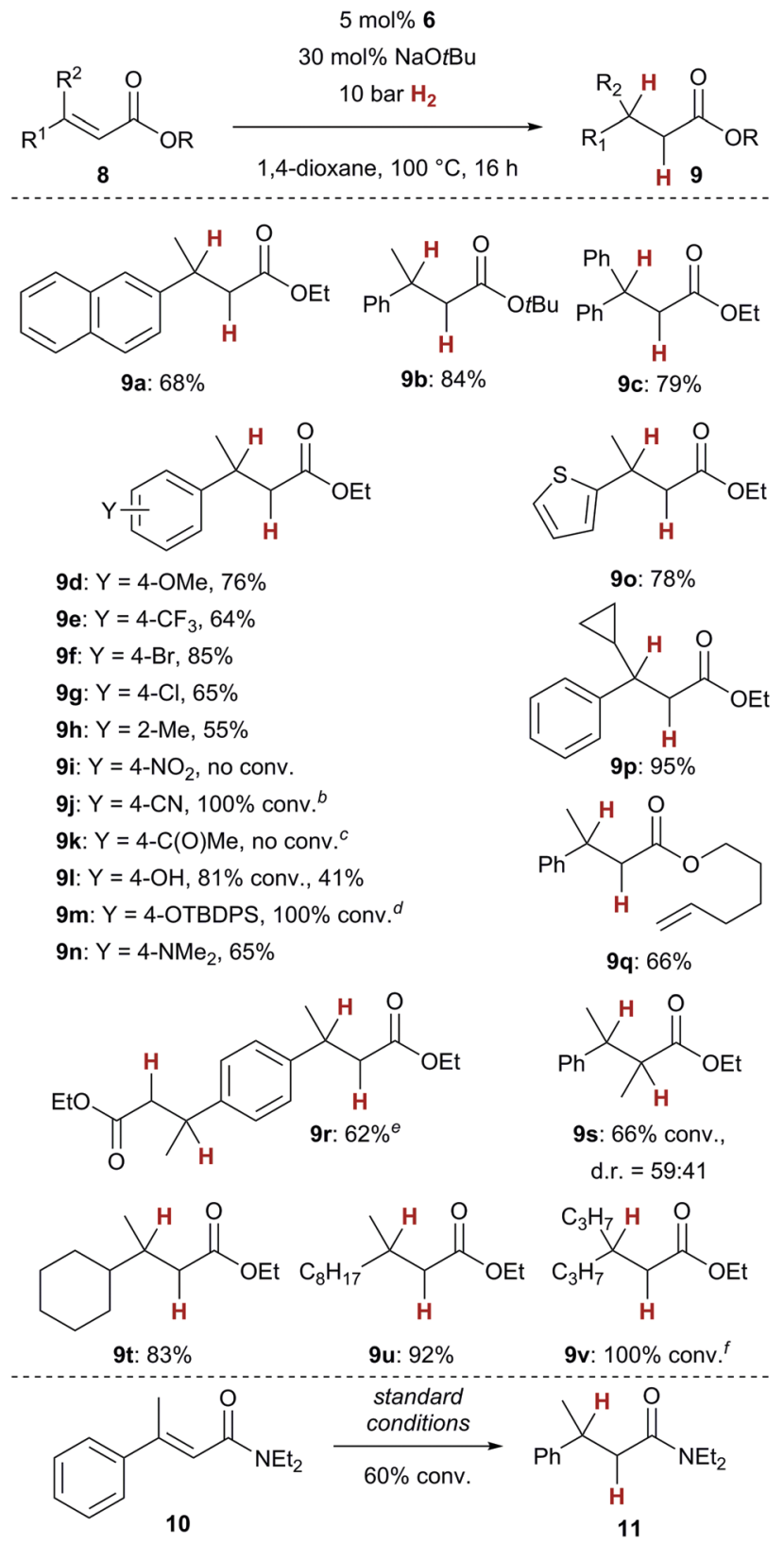

Scheme 2 Cu-catalysed hydrogenation of enoates and enamides, scope . $^{a}$ ${ }^{a}$ If not noted otherwise, $E$-configured enoates $\mathbf{8}$ have been employed. $E / Z$ ratios: $\mathbf{8 f}: 90: 10, \mathbf{8 i}: 88: 12, \mathbf{8 k}: 87: 13, \mathbf{8 m}: 93: 7, \mathbf{8 u}: 77: 23{ }^{b}$ The product decomposed during purification, $5 \%$ isolated yield. ${ }^{C}$ No 1,2 -reduction of the ketone was observed. ${ }^{d} A$ mixture of the corresponding ethyl and tert-butyl ester were observed $(83: 17)$. The separation turned out to be tedious, resulting in lower yields (OEt: 40\%, OtBu: 7\%). ${ }^{e} 10 \mathrm{~mol} \%$ 6, $60 \mathrm{~mol} \% \mathrm{NaOtBu}$ used. ${ }^{f}$ Product is volatile.

conjugate reduction. ${ }^{14}$ Also, the silyl-protected variant (9m) could be converted successfully. Dimethylaniline 9n and thiophene-derived 90 as possibly coordinating substrates could be successfully hydrogenated. The generation of cyclopropanesubstituted 9p in almost quantitative yield (95\%) gives an important indication that no carbon-based radical is involved in the overall process. The clean formation of alkene-substituted ester 9q underscores the chemoselectivity of the present catalyst, 
as no alkene hydrogenation was observed..$^{15}$ Next to the successful formation of diester 9r, also 9s, bearing a methyl group in $\alpha$-position, which generally slows down the conjugate addition in other copper-catalysed processes, ${ }^{16}$ was turned over by the catalyst. Ester 9s was formed as a 59:41 mixture of diastereomers. Finally, dialkyl-substituted enoates $\mathbf{9 t}-\mathbf{9 v}$ could be converted with similarly good results in terms of yield. We could demonstrate that even enamide 10, which generally is too electron-rich for reactivity in other copper-catalysed conjugate addition reactions, ${ }^{17}$ displays some reactivity with our catalyst (60\% conv., $17 \%$ yield for 11 ). This result underscores the fact that the copper/NHC complexes employed in this transformation serve a key role for the generation of copper hydride intermediates with higher reactivity in comparison to the commonly used phosphine complexes. ${ }^{5}$ The latter could not realize any conversion of carboxylic acid derivatives.

Sorbic acid derivative 12 gave a mixture of 1,4- and 1,6-addition products, with unsaturated ester 13a (from 1,4-addition of the hydride nucleophile) as the major product (Scheme 3). The formation of 13b and 13c can be explained by prior 1,6-addition of the copper hydride, and, in the case of 13c, subsequent 1,4-addition.

To gain some insight into the mechanism of the present protocol, we carried out the conjugate reduction in the presence of deuterium gas $\left(D_{2}\right.$, Scheme 4$)$. With ethyl esters $\mathbf{8 c}$ and $\mathbf{8 d}$, as expected for a hydride transfer reaction, the deuterium incorporation in the $\beta$ position was high $(\geq 90 \% \mathrm{D})$, but also significant isotope labeling in the $\alpha$ position was found, indicating an enolisation process with $t$-BuOD after the conjugate addition. Unexpectedly, also deuterium incorporation was observed in the ethyl ester $\left(23-39 \% \mathrm{D}, \mathrm{D}_{1^{\prime}}\right.$ and $\left.\mathrm{D}_{2^{\prime}}\right){ }^{18}$

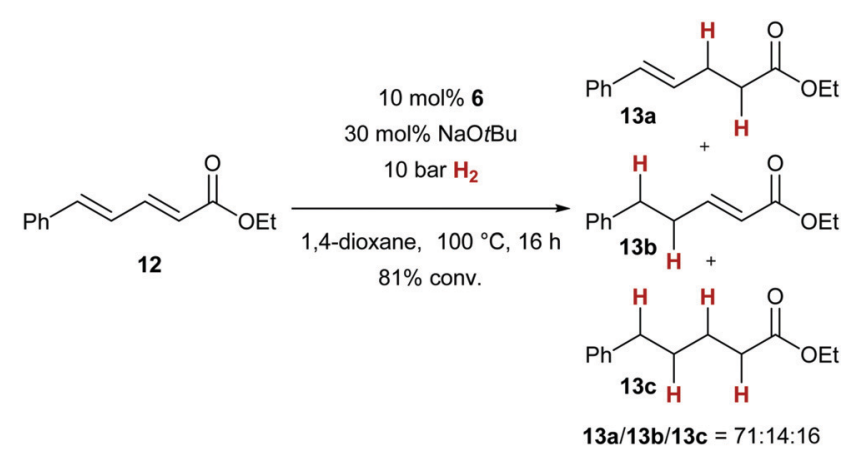

Scheme 3 Conjugate reduction of an $\alpha, \beta, \gamma, \delta$-unsaturated ester.

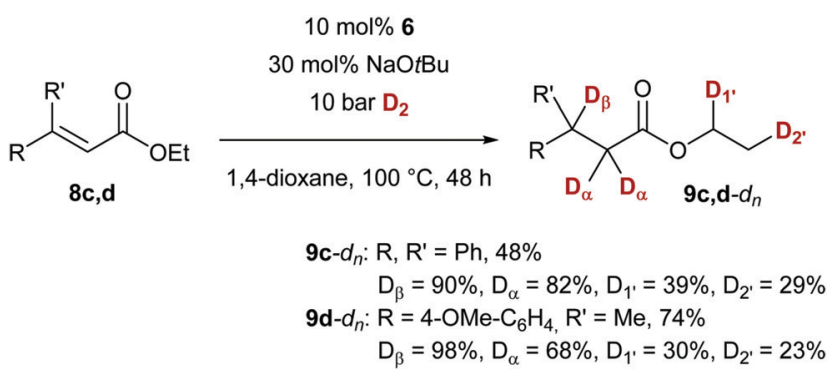

Scheme 4 Cu-catalysed conjugate reduction with $D_{2}$.

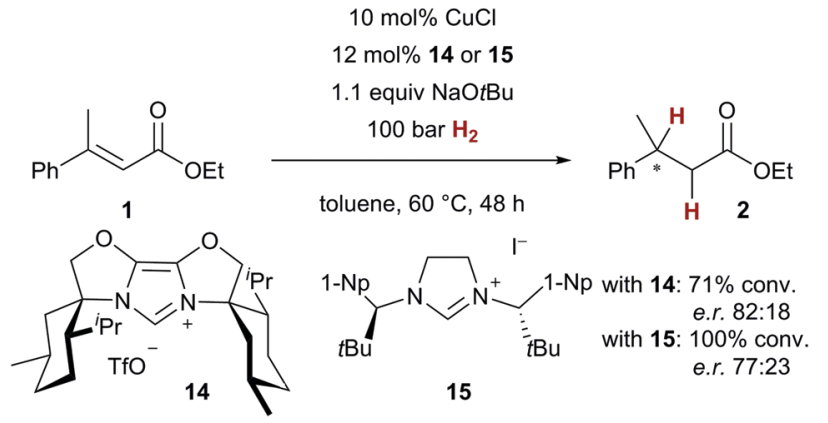

Scheme 5 Asymmetric conjugate reduction of cinnamate $1(1-\mathrm{Np}=$ 1-naphthyl).

As an asymmetric variant of catalytic conjugate reduction with $\mathrm{H}_{2}$ is highly attractive, ${ }^{16}$ we turned our attention to chiral NHC ligands. In preliminary experiments, we were able to demonstrate that indeed stereoinduction with chiral NHC ligands is possible, even though more drastic reaction conditions (100 bar $\mathrm{H}_{2}, 48 \mathrm{~h}$ reaction time) had to be employed with chiral NHC precursors $\mathbf{1 4}^{19}$ and $\mathbf{1 5}^{20}$ (Scheme 5). The corresponding reduced ester 2 was obtained with an enantiomeric ratio of up to $82: 18$ with chiral NHC precursor 14, demonstrating the viability of an asymmetric reaction.

In summary, we have developed a simple and efficient copper-catalysed conjugate reduction of enoates and enamides with $\mathrm{H}_{2}$. Well-defined and easily accessible copper(I)/NHC complexes are employed as catalysts, giving the desired products which had so far not been accessible with copper/phosphine complexes due to the lower reactivity of these substrates employing $\mathrm{H}_{2}$. The present catalytic protocol serves as an atom economic alternative to replace the commonly used and waste-generating hydrosilanes as stoichiometric reducing agents with dihydrogen $\left(\mathrm{H}_{2}\right)$. Isotope labelling studies indicate a polar mechanism involving a copper hydride intermediate. In addition, the viability of an asymmetric reaction has been demonstrated.

This work was supported by the German Research Council (DFG, Emmy Noether Fellowship for J. F. T., TE1101/2-1), by the Fonds der Chemischen Industrie (Liebig-Stipendium for J. F. T.) and by the Daimler and Benz Foundation (postdoctoral fellowship for J. F. T.). Prof. Dr Martin Oestreich (TU Berlin) is kindly thanked for generous support.

\section{Conflicts of interest}

There are no conflicts to declare.

\section{Notes and references}

1 For a review on green chemistry, see: P. Anastas and N. Eghbali, Chem. Soc. Rev., 2010, 39, 301-312.

2 For a reviews on atom economy and atom efficiency, see: $(a)$ R. A. Sheldon, Chem. Soc. Rev., 2012, 41, 1437-1451; (b) B. M. Trost, Angew. Chem., Int. Ed., 1995, 34, 259-281.

3 The handbook of homogeneous hydrogenation, ed. J. G. de Vries and C. J. Elsevier, Wiley-VCH, Weinheim, 2007.

4 The use of $\mathrm{H}_{2}$ as reagent has been put forward, see for example: (a) R. Noyorii, Chem. Commun., 2005, 1807-1811; (b) D. J. C. Constable, P. J. Dunn, J. D. Hayler, G. R. Humphrey, J. J. L. Leazer, R. J. Linderman, 
K. Lorenz, J. Manley, B. A. Pearlman, A. Wells, A. Zaks and T. Y. Zhang, Green Chem., 2007, 9, 411-420.

5 For reviews, see: (a) A. J. Jordan, G. Lalic and J. P. Sadighi, Chem. Rev., 2016, 116, 8318-8372; (b) B. H. Lipshutz, in Copper-catalysed asymmetric synthesis, ed. A. Alexakis, N. Krause and S. Woodward, Wiley-VCH, Weinheim, 2014, vol. 110, pp. 179-202; (c) C. Deutsch, B. H. Liphsutz and N. Krause, Chem. Rev., 2008, 108, 2916-2927; (d) S. Rendler and M. Oestreich, Angew. Chem., Int. Ed., 2007, 46, 498-504.

6 For the catalytic hydrogenation of enones, enals and carbonyl compounds, see: (a) W. S. Mahoney and J. M. Stryker, J. Am. Chem. Soc., 1989, 111, 8818-8823; (b) J.-X. Chen, J. F. Daeuble, D. M. Brestensky and J. M. Stryker, Tetrahedron, 2000, 56, 2153-2166; (c) J.-X. Chen, J. F. Daeuble and J. M. Stryker, Tetrahedron, 2000, 56, 2789-2798; (d) H. Shimizu, D. Igarashi, W. Kuriyama, Y. Yusa, N. Sayo and T. Saito, Org. Lett., 2007, 9, 1655-1657; (e) H. Shimizu, N. Sayo and T. Saito, Synlett, 2009, 1295-1298; $(f)$ H. Shimizu, T. Nagano, N. Sayo, T. Saito, T. Ohshima and K. Mashima, Synlett, 2009, 3143-3146; $(g)$ K. Junge, B. Wendt, D. Addis, S. Zhou, S. Das, S. Fleischer and M. Beller, Chem. - Eur. J., 2011, 17, 101-105.

7 For copper-catalysed semihydrogenations of alkynes, see: (a) K. Semba, R. Kameyama and Y. Nakao, Synlett, 2015, 318-322; (b) F. Pape, N. O. Thiel and J. F. Teichert, Chem. - Eur. J., 2015, 21, 15934-15938; (c) N. O. Thiel and J. F. Teichert, Org. Biomol. Chem., 2016, 14, 10660-10666; (d) T. Wakamatsu, K. Nagao, H. Ohmiya and M. Sawamura, Organometallics, 2016, 35, 1354-1357; (e) F. Pape and J. F. Teichert, Synthesis, 2017, 2470-2482; $(f)$ N. O. Thiel, S. Kemper and J. F. Teichert, Tetrahedron, 2017, 73, 5023-5028.

8 This reactivity trend was also observed employing hydrosilanes, see: V. Jurkauskas, J. P. Sadighi and S. L. Buchwald, Org. Lett., 2003, 5, 2417-2420.

9 For a recently published report on a related heterogeneous hydrogenation of $\alpha, \beta$-unsaturated carbonyl compounds, see: J. Mendes-Burak, B. Ghaffari and C. Copéret, Chem. Commun., 2019, 55, 179-181.
10 J. F. Hartwig, Organotransition metal chemistry, From bonding to catalysis, University Science Books, Mill Valley, 2010.

11 For reviews, see: (a) F. Lazreg, F. Nahra and C. S. J. Cazin, Coord. Chem. Rev., 2015, 293-294, 48-79; (b) F. Lazreg and C. S. J. Cazin, in $\mathrm{N}$-Heterocyclic carbenes. Effective tools for organometallic synthesis, ed. S. P. Nolan, Wiley-VCH, Weinheim, 2014, vol. 113, pp. 199-242.

12 (a) A. J. Chalk and J. Halpern, J. Am. Chem. Soc., 1959, 81, 5852-5854; (b) J. Halpern, J. Phys. Chem., 1959, 63, 398-403; (c) G. V. Goeden and K. G. Caulton, J. Am. Chem. Soc., 1981, 103, 7354-7355.

13 See the ESI $\dagger$ for details.

14 For investigation of further protic additives, see the ESI†.

15 The copper-catalyzed alkyne semihydrogenation (compare ref. 7) is competitive, see the ESI $\dagger$.

16 For reviews, see: (a) T. Jerphagnon, M. G. Pizzuti, A. J. Minnaard and B. L. Feringa, Chem. Soc. Rev., 2009, 38, 1039-1075; (b) S. R. Harutyunyan, T. den Hartog, K. Geurts, A. J. Minnaard and B. L. Feringa, Chem. Rev., 2008, 108, 2824-2852; (c) A. Alexakis, J. E. Bäckvall, N. Krause, O. Pàmies and M. Diéguez, Chem. Rev., 2008, 108, 2796-2823.

17 For a notable exception, see: M. Rodríguez-Fernández, X. Yan, J. F. Collados, P. B. White and S. R. Harutyunyan, J. Am. Chem. Soc., 2017, 139, 14224-14231.

18 The mechanism of the $\mathrm{D}$ incorporation of the ester is unclear at present. Experiments also show D incorporation in the ester moeity when the reduced ester 2 is submitted to the standard conditions employing $D_{2}$. Further studies in shedding light on this process are currently underway. At present, a second, heterogenous process cannot be excluded. For the distinction between homogeneous and heterogenous catalysis, see: (a) R. H. Crabtree, Chem. Rev., 2012, 112, 1536-1554; (b) J. A. Widegren and R. G. Finke, J. Mol. Catal. A: Chem., 2003, 198, 317-341; (c) ref. 9.

19 G. Altenhoff, R. Goddard, C. W. Lehmann and F. Glorius, J. Am. Chem. Soc., 2004, 126, 15195-15201.

20 D. Katayev, Y.-X. Jia, A. K. Sharma, D. Banerjee, C. Besnard, R. B. Sunoj and E. P. Kündig, Chem. - Eur. J., 2013, 19, 11916-11927. 